\title{
Re-Shielding of Cobalt-60 Teletherapy Rooms for Tomotherapy and Conventional Linear Accelerators using Monte Carlo Simulations
}

\author{
Yiğit Çeçen ${ }^{1,2, a}$, Çağrı Yazgan ${ }^{1,2}$ \\ ${ }^{1}$ Akdeniz University, Nuclear Sciences Application and Research Center, 07058, Antalya, Turkey \\ ${ }^{2}$ Akdeniz University, School of Medicine, Department of Radiation Oncology, 07058, Antalya, Turkey
}

\begin{abstract}
Purpose. Nearly all Cobalt-60 teletherapy machines were removed around the world during the last two decades. The remaining ones are being used for experimental purposes. However, the rooms of these teletherapy machines are valuable because of lack of space in radiotherapy clinics. In order to place a new technology treatment machine in one of these rooms, one should re-shield the room since it was designed only for $1.25 \mathrm{MeV}$ gamma beams on average. Mostly, the vendor of the new machine constructs the new shielding of the room using their experience. However, every radiotherapy room has different surrounding work areas and it would be wise to shield the room considering these special conditions. Also, the shield design goal of the clinic may be much lower than the International Atomic Energy Agency (IAEA) or the local association accepts. The study shows re-shielding of a Cobalt-60 room, specific to the clinic, using Monte Carlo simulations.
\end{abstract}

Materials \& Methods. First, a 6 MV Tomotherapy machine, then a 10 MV conventional linear accelerator (LINAC) was placed inside the Cobalt-60 teletherapy room. The photon flux outside the room was simulated using Monte Carlo N-Particle (MCNP6.1) code before and after re-shielding.

For the Tomotherapy simulation, flux distributions around the machine were obtained from the vendor and implemented as the source of the model. The LINAC model was more generic with the $10 \mathrm{MeV}$ electron source, the tungsten target, first and secondary collimators. The aim of the model was to obtain the maximum $\left(40 \mathrm{x} 40 \mathrm{~cm}^{2}\right)$ open field at the isocenter. Two different simulations were carried out for gantry angles $90^{\circ}$ and $270^{\circ}$. The LINAC was placed in the room such that the primary walls were $A^{\prime}\left(\right.$ Gantry $\left.270^{\circ}\right)$ and $C^{\prime}\left(\right.$ Gantry $\left.90^{\circ}\right)$ (figure 1 ).

The second part of the study was to model the re-shielding of the room for Tomotherapy and for the conventional LINAC, separately. The aim was to investigate the recommended shielding by the vendors. Left side of the room was adjacent to a LINAC room with 2 meters thick concrete wall (figure 1). No shielding was necessary for that wall. Behind wall A-A' there was an outdoors forbidden area; behind wall B-B' was the contouring room for the doctors; and the control room was behind wall C-C' (figure 1). After some modifications, the final shielding was designed.

Results. The photon flux distributions outside the room before and after the re-shielding were compared. The reshielding of Tomotherapy reduced the flux down to $1.89 \%$ on average with respect to pre-shielding (table 1). For the conventional LINAC case; after re-shielding, the photon flux in the control room -which corresponds to gantry $90^{\circ}$ decreased down to $0.57 \%$ with respect to pre-shielding (table 2). The photon flux behind wall A' -which corresponds to gantry $270^{\circ}$ - decreased down to $2.46 \%$. Everybody was all safe behind wall B' even before re-shielding.

\footnotetext{
${ }^{\mathrm{a}}$ Corresponding author: ycecen@yahoo.com
} 
Table 1. Highest photon fluxes behind the walls for the Tomotherapy model before and after re-shielding.

\begin{tabular}{|c|c|c|c|c|c|}
\hline Wall & $\begin{array}{c}\text { Relative Photon Flux } \\
\text { Before re-shielding }\end{array}$ & $\begin{array}{c}\text { Relative } \\
\text { Error (\%) }\end{array}$ & $\begin{array}{c}\text { Relative Photon Flux } \\
\text { After re-shielding }\end{array}$ & $\begin{array}{c}\text { Relative } \\
\text { Error (\%) }\end{array}$ & $\begin{array}{c}\text { Decrease in } \\
\text { photon flux (\%) ) }\end{array}$ \\
\hline A & $2.71 \mathrm{E}-06$ & 0.24 & $5.74 \mathrm{E}-08$ & 0.42 & 97.88 \\
\hline B & $6.94 \mathrm{E}-06$ & 0.31 & $1.11 \mathrm{E}-07$ & 0.29 & 98.41 \\
\hline C & $2.86 \mathrm{E}-06$ & 0.67 & $5.11 \mathrm{E}-08$ & 0.37 & 98.21 \\
\hline
\end{tabular}

Table 2. Highest photon fluxes behind the walls for the conventional LINAC model before and after re-shielding.

\begin{tabular}{|c|c|c|c|c|c|}
\hline Wall & $\begin{array}{c}\text { Relative Photon Flux } \\
\text { Before re-shielding }\end{array}$ & $\begin{array}{c}\text { Relative } \\
\text { Error (\%) }\end{array}$ & $\begin{array}{c}\text { Relative Photon Flux } \\
\text { After re-shielding }\end{array}$ & $\begin{array}{c}\text { Relative } \\
\text { Error (\%) }\end{array}$ & $\begin{array}{c}\text { Decrease in } \\
\text { photon flux (\%) }\end{array}$ \\
\hline A' $^{\prime}\left(\mathbf{G 2 7 0}^{\mathbf{}}\right)$ & $1,81 \mathrm{E}-05$ & 0.54 & $4,45 \mathrm{E}-07$ & 0.34 & 97,54 \\
\hline $\mathbf{B}^{\prime}$ & 0 & 0 & 0 & 0 & 0 \\
\hline $\mathbf{C}^{\prime}\left(\mathbf{G 9 0}^{\mathbf{0}}\right)$ & $2,31 \mathrm{E}-05$ & 0.59 & $1,31 \mathrm{E}-07$ & 0.29 & 99,43 \\
\hline
\end{tabular}

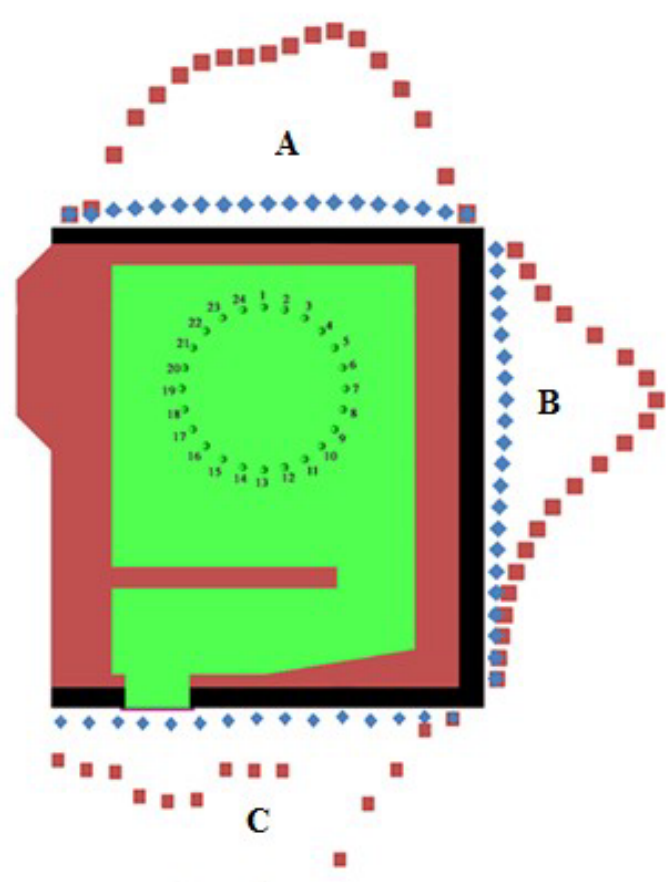

Tomotherapy

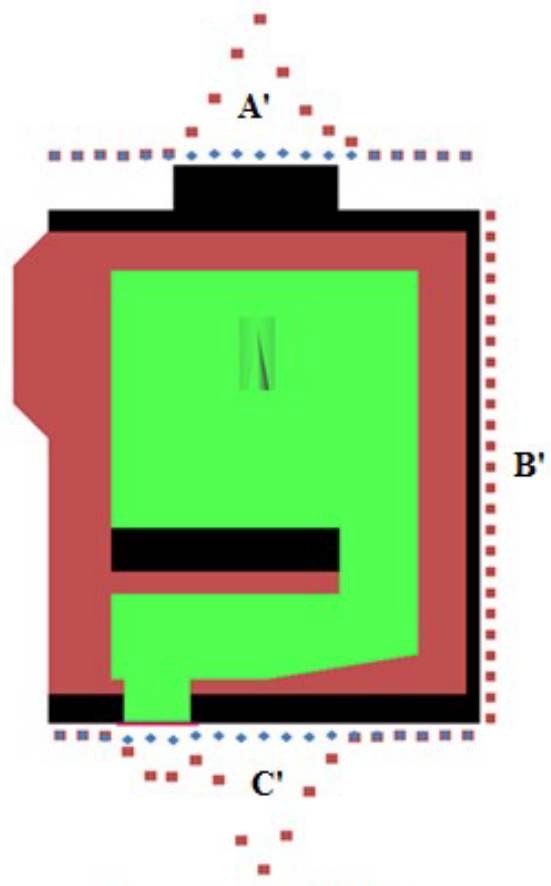

Conventional LINAC

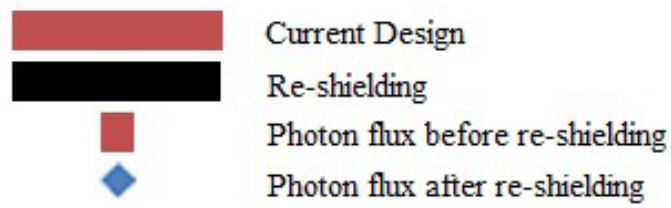

Figure 1. The photon flux distributions outside the Cobalt-60 room before and after re-shielding. 
Conclusion. This study assumes that flux and dose relation is linear and 1 Gy dose corresponds to the flux at the isocenter for the LINAC case. For the Tomotherapy case, the dose distributions were given along with the flux distributions by the vendor. The fluxes calculated by the Monte Carlo simulations should give a hint for the instantaneous dose rates (IDR). More work is needed to calibrate the findings with the design goals and achieve correct comparison for the dose behind the walls.

In order to place new technology treatment machines inside old Cobalt-60 teletherapy rooms which were designed to shield only $1.25 \mathrm{MeV}$ gamma radiation, it is necessary to design radiation shielding for high energy photons. However, the re-shielding should not solely be left to the vendor's experience. The re-shielding calculations should be made according to International-National Committee on Radiation Protection (ICRP-NCRP) protocols and, if possible, Monte Carlo simulations should be used specifically to meet the clinics needs. 